\title{
Molecular diagnosis of McArdle disease using whole-exome sequencing
}

\author{
JU-HYUNG KANG $^{1 *}$, JUN-HYUNG PARK ${ }^{2 *}$, JIN-SOON PARK $^{2}$, \\ SEONG-KYU LEE ${ }^{2}$, SUNGHOON LEE ${ }^{3}$ and HAING-WOON BAIK ${ }^{2}$
}

Departments of ${ }^{1}$ Pediatrics and ${ }^{2}$ Biochemistry and Molecular Biology, School of Medicine, Eulji University, Daejeon 34824; ${ }^{3}$ Department of Research and Development Eone-Diagnomics Genome Center, Incheon 22014, Republic of Korea

Received November 19, 2020; Accepted April 28, 2021

DOI: $10.3892 /$ etm.2021.10461

\begin{abstract}
Whole-exome sequencing (WES) analysis has been used recently as a diagnostic tool for finding molecular defects. In the present study, researchers attempted to analyze molecular defects through WES in a 13-year-old female patient who had not been diagnosed through a conventional genetic approach. DNA was extracted and subjected to WES analysis to identify the genetic defect. A total of 106,728 exons and splicing variants were selected, and synonymous single nucleotide variants (SNVs) and general single nucleotide polymorphisms (SNPs) were filtered out. Finally, nonsynonymous SNVs (c.C415T and c.C389T) of the PYGM gene were identified in nine compound heterozygous mutations. PYGM encodes myophosphorylase and degrades glycogen in the muscle to supply energy to muscle cells. The present study revealed that the patient's father had a c.C389T mutation and the mother had a c.C415T mutation, resulting in A130V and R139W missense mutations, respectively. To the best of our knowledge, the A130V variant in PYGM has not been reported in the common variant databases. All variations of the patient's family detected using WES were verified by Sanger sequencing. Because the patient had compound heterozygous mutations in the PYGM gene, the patient was presumed to exhibit markedly decreased muscle phosphorylase activity. To assess the function of myophosphorylase, an ischemic forearm exercise test was performed. The blood ammonia level sharply increased and the lactate level maintained a flat curve shape similar to the typical pattern of McArdle disease. Therefore, the diagnosis of the patient was confirmed to be McArdle disease, a glycogen storage disease. Through WES analysis,
\end{abstract}

Correspondence to: Professor Haing-Woon Baik, Department of Biochemistry and Molecular Biology, School of Medicine, Eulji University, 77 Gyeryong-ro 771 Gil, Daejeon 34824, Republic of Korea

E-mail: baikhw@eulji.ac.kr

*Contributed equally

Key words: whole-exome sequencing, McArdle disease, PYGM accurate and early diagnosis could be made in the present study. This report describes a novel compound heterozygous mutation of the PYGM gene in a Korean patient.

\section{Introduction}

McArdle disease is a glycogen storage disease, a rare genetic disorder caused by deficient myophosphorylase activity. Myophosphorylase is a key enzyme in energy metabolism because it catalyzes the rate-limiting step in glycogenolysis in muscle (1). Glycogen phosphorylase has three isoforms: PYGB (brain), PYGL (liver) and PYGM (muscle). It has been reported that PYGM is present in skeletal muscle as well as in other tissues and organs. The role of PYGM in tissues other than muscle is unknown, but it is presumed to play a role in not only McArdle disease but also in other diseases (2). McArdle disease is caused by genetic defects of the myophosphorylase enzyme gene, PYGM, and the disease has an autosomal recessive inheritance (3). More than 150 human PYGM mutations have been identified, and the most common genetic defect in Caucasians with McArdle disease is the nonsense mutation in p.R50X of exon 1 (4). Although McArdle disease occurs in about 1 in 100,000 people in the United States and Europe, it is very rare in Korea, showing racial differences (5-7). The p.R50X mutation accounts for more than 50\% of PYGM mutations in patients with McArdle disease in Europe, and only one case has been reported in Japan (5). Therefore, it has been suggested that there are ethnic differences in PYGM variation between European and Asian individuals. Furthermore, $54 \%$ of PYGM mutations were homozygous and the rest were heterozygous allele $(8,9)$. Patients with McArdle disease experience progressive weakness and muscle wasting, and some patients may not be diagnosed with this disease until they are over 50 years of age because they have no apparent symptoms (10). In patients with McArdle disease, starting from the age of 50, symptoms of fixed muscle weakness around the shoulder appear and may be accompanied by cardiomyopathy, respiratory failure, and nervous system disorders $(1,11)$.

Recently, whole-exome sequencing (WES) has proved to be a very effective method for the detection of familial disease genes and a reliable methodology for detecting and quantifying genetic defects clinically. Next-generation sequencing has made a number of technological advances with minimal validation 
errors and reliable levels of specificity and sensitivity (12). WES is a diagnostic approach for detecting molecular defects in patients with suspected genetic diseases (13). In the present study, we attempted to analyze molecular defects through WES in a 13-year-old female patient who had not been diagnosed through a conventional genetic approach. This study identified compound heterozygous mutations of $P Y G M$, which were found to be new disease-causing missense mutations, in a Korean family by WES.

\section{Subjects and methods}

Subjects. A 13-year-old female patient visited the Eulji University Hospital Growth Clinic because of her short stature (3rd percentile). At first visit, she presented poor weight gain (1st percentile) together and muscle weakness and dyspnea developed immediately after short term exercise. At past history, she had administered at hospital for 1 month a few years before because of general ache and muscle weakness developed by excessive physical activity. At first, we performed routine laboratory tests including growth hormone stimulation to investigate the etiology of short stature and poor weight gain but there were no remarkable findings. We planned a genetic test for the patient, and the PYGM mutation was discovered through WES, which brought attention to the clinical features associated with myophosphorylase deficiency. During the period of regular follow up, the patient showed exercise intolerance and the second wind phenomenon. She was in the fifth grade of elementary school, and her intelligence was normal. The patient's parents and the younger sister, who was one year younger than the patient, had no medically specific findings (Fig. 1). The present study was approved by the Institutional Review Board of Eulji University (approval no. EU17-42; approval date, December 14, 2017), and written informed consent was obtained from all individual participants included in this study; for participants $<18$ years old, the patients' parents provided written informed consent. The patient's family has provided written informed consent for publication.

Genomic DNA extraction. Genomic DNA was extracted from the blood of the patient and all of her family members, and the gDNA was quantitatively analyzed and confirmed by agarose gel electrophoresis.

WES analysis. The entire exome sequencing process was performed in three stages: Sample preprocessing and sequencing, primary data processing, and secondary data processing. First, genomic DNA was fragmented and reconnected to produce a library for sequencing. Exome capture and enrichment were performed using a human exome capture kit (Illumina). WES was performed using high-throughput nextgeneration sequencing (NGS) technology in the Illumina GA/ HiSeq 2000 sequencing system (Illumina).

Genomic DNA was extracted using AccuPrep ${ }^{\circledR}$ Genomic DNA Extraction kit (Bioneer) to prepare DNA samples for sequencing. To generate standard exome capture libraries, SureSelectXT Reagent kits (Agilent) were used. The DNA quantity and quality were measured by Quant-iT ${ }^{\mathrm{TM}}$ PicoGreen $^{\mathrm{TM}}$ dsDNA assay kit, (Cat. \# P7589, Thermo Fisher Scientific, Inc.) and agarose gel electrophoresis,

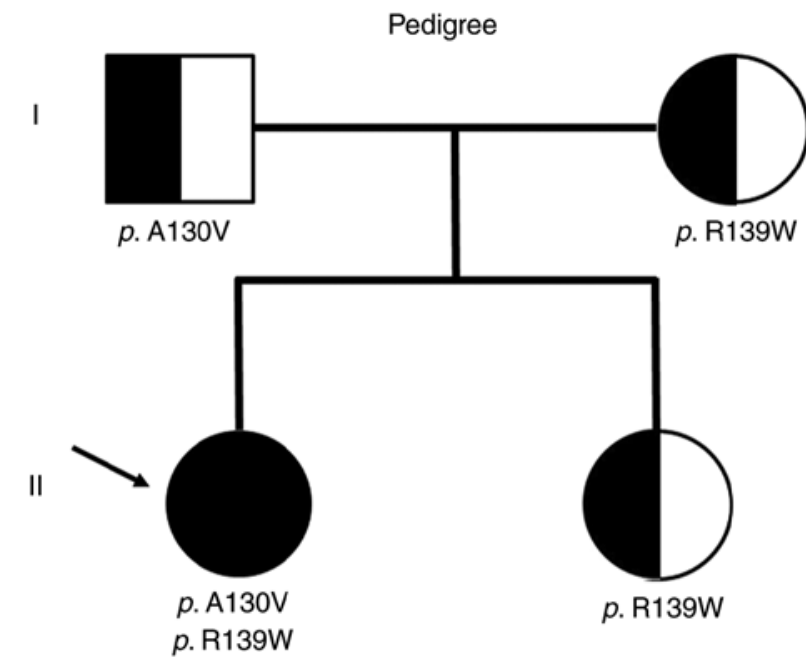

Figure 1. Pedigree of the patient with mutations in the PYGM gene. Two missense mutations were observed in the study: p.A130V, alanine to valine at residue 130 in PYGM; p.R139W, arginine to tryptophan at residue 139 in PYGM. Squares, male; circles, female; filled symbol, affected; half-black symbol, heterozygous for recessive allele. Arrow indicates the proband.

respectively. One microgram of each cell line's genomic DNA was diluted with EB Buffer and sheared to a target peak size of 150-200 bp with the Covaris LE220 focused-ultrasonicator (Covaris) according to the manufacturer's recommendations. Fragmentation was followed by end-repair and the addition of an 'A' tail. Then Agilent adapters were ligated to the fragments. After evaluating the efficiency of ligation, the adapter ligated product was polymerase chain reaction (PCR) amplified. The final purified product was quantified by TapeStation DNA screentape D1000 (Agilent). For exome capture, $250 \mathrm{ng}$ of DNA library was mixed with hybridization buffers, blocking mixes, RNase block and $5 \mu$ l of SureSelect all exon capture library, according to the standard Agilent SureSelect Target Enrichment protocol. The captured DNA was washed and amplified. Then final purified product was quantified by qPCR according to the qPCR Quantification Protocol Guide (KAPA Library Quantification kits for Illumina Sequencing platforms) and qualified by the TapeStation DNA screentape D1000 (Agilent). The concentration of the final library was 0.228 to 0.304 pmol, using PicoGreen dsDNA Quantitation Reagent (Promega). NovaSeq 6000 S4 Reagent Kit v1.5 (Cat. \# 20028312, Illumina) was used for sequencing. When analyzing the data obtained through WES, BWA (http:// biobwa.sourceforge.net/bwa.shtml), Picard (http://broadinstitute.github.io/picard), SnpEff, and GATK (https://www. broadinstitute.org/gatk) software were used.

An average of thirty times was considered sufficient for accurate mutation detection. The data obtained from the NGS platform were passed to raw sequence reads and processed. These reads were aligned to the reference genome using the alignment tool. When noise was generated in variant calling, PCR copies were removed. In the subsequent analysis, variant calling and filtering were performed to eliminate false positives according to quality control standards. Finally, before testing to identify mutations that cause disease, mutations were identified to obtain information on gene location and action effects. 


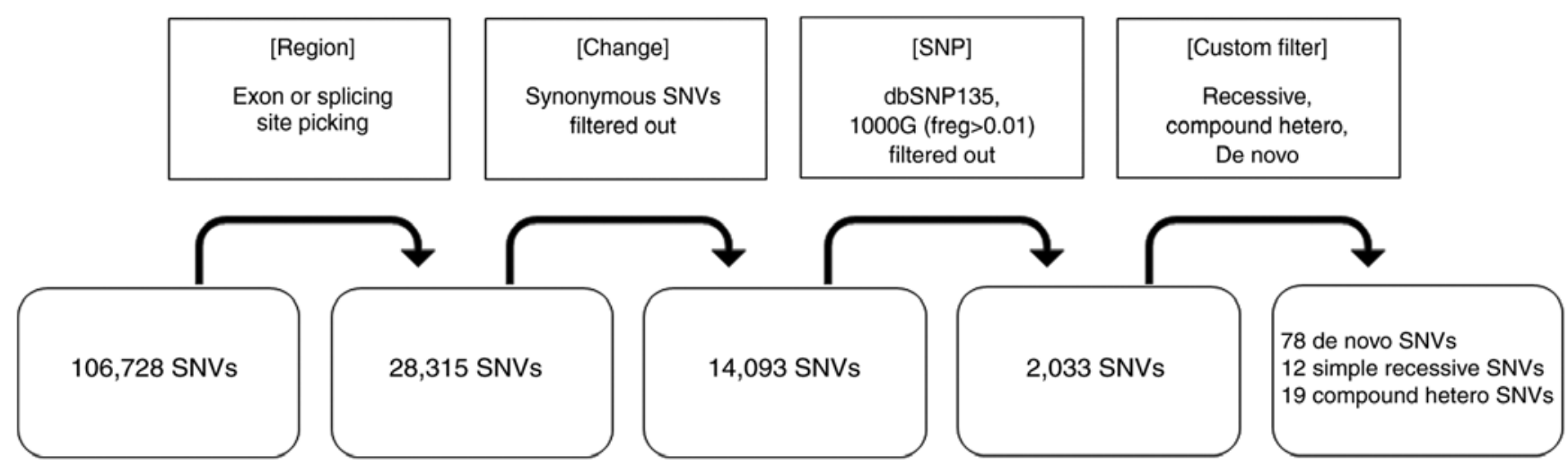

Figure 2. Workflow of data analysis. The raw data resulting from Whole-Exome Sequencing was summarized. A total of 106,728 variations were detected in the patient by WES. As a result of limiting the mutation sites to exon and splicing sites, variation was reduced to 28,315 , and the number was further reduced to 14,093 by filtering out synonymous SNVs. As a result of applying the recessive, compound heterozygous, and de novo conditions to the remaining 2,033 variations by filtering under the conditions of dbSNP135_common $1000 \mathrm{G}$ (freq. > 0.01), 78 de novo mutations, 12 simple recessive mutations, and 9 compound heterozygous mutations were isolated. SNV, single-nucleotide variant; SNP, single-nucleotide polymorphism.

Table I. List of 12 homozygous recessive genes of the patient.

\begin{tabular}{llll}
\hline Gene & Polymorphic change & \multicolumn{1}{c}{ Function } & \multicolumn{1}{c}{ Related disease } \\
\hline ALLC & Non-frameshift deletion & Purine metabolism & Lymphatic granuloma \\
FAM171B & Non-frameshift insertion & Expression of polyglutamine region & Polyglutamine disease \\
HYAL1 & Nonsynonymous SNV & Hyaluronic acid (HA) & HA deficiency \\
$T C P 11$ & Frameshift deletion & Spermatogenesis & Abnormal sperm \\
TMEM184A & Non-frameshift insertion & Expression in testis & Abnormal sperm \\
AHR & Nonsynonymous SNV & Regulation of metabolic enzymes & Abnormal xenobiotics metabolism \\
LRRC17 & Frameshift deletion & Regulation of osteoclast & Overdifferentiation of osteoclast \\
PTCHD3 & Frameshift insertion & Spermatozoa function & Abnormal sperm \\
SPRN & Non-frameshift deletion & Neuroprotection & Neuroprotection associated disease \\
OR4L1 & Frameshift deletion & Olfaction & Olfactory weakness \\
ZFHX3 & Non-frameshift deletion & Differentiation of muscle and nerve & Abnormal muscle and nerve differentiation \\
KRTAP17-1 & Non-frameshift deletion & High sulfur-associated protein & High Sulfur associated \\
\hline
\end{tabular}

SNV, single-nucleotide variant.

PCR and Sanger sequencing. PCR was performed using AccuPower ${ }^{\circledR}$ PreMix (Bioneer) for sequence analysis of mutations in the 20 exons of the PYGM gene. Two sets of primers were prepared, and nested PCR was performed to obtain a clean PCR product without nonspecific reactions. The amplified PCR products were purified and subjected to dideoxy-sequencing PCR and purification, followed by sequencing using a 3730XL DNA analyzer (Thermo Fisher Scientific Inc.).

Forearm exercise test. The forearm exercise test was performed using the method developed by Kazemi-Esfarjani et al (14). Before the test, $0.5 \mathrm{ml}$ of venous blood was sampled to determine the amounts of ammonia and lactic acid. A blood pressure measurement cuff was wrapped around the right upper arm, and the patient was held at an interval of $3 \mathrm{sec}$ while air was injected into the cuff over the systolic period. At this time, hand-grasping exercises were performed just until muscle pain appeared, and the fingers could be completely bent and unfolded. Antecubital venous blood samples were taken at $1,2,3$, and 5 min after the cuff air was removed. After that, the patient had difficulty in hand movement for about $2 \mathrm{~min}$, remarkable muscle buildup was observed in the forearm, and the fingers could not be spread. The concentrations of ammonia and lactic acid were measured in the patient's blood taken at each time point.

\section{Results}

Variant filtering and gene classification. The patient's wholeexome DNA was analyzed by library production, sequencing, and data sorting. The quality score and nucleotide distribution for NGS were in the normal range, the DNA fragment inserted into the library was 100-200 bp in size, and the depth distribution was in the normal range. The raw data resulting from WES are summarized in Fig. 2. A total of 106,728 variations were detected in the patient by WES. As a result of limiting the mutation sites to exon and splicing sites, variation 
Table II. List of nine compound heterozygous recessive genes of the patient.

\begin{tabular}{llll}
\hline Gene & Polymorphic change & & Function \\
\hline CYP4A22 & Nonsynonymous SNV & Cytochrome P450 & Abnormal cyt P450 \\
ZNF644 & Nonsynonymous SNV & Eye development & High myopia \\
SYNJ2 & Nonsynonymous SNV & PIP activity & Charcot-Marie-Tooth disease \\
NOL8 & Nonsynonymous SNV & Cell growth & Abnormal growth \\
AHNAK & Nonsynonymous SNV & Cell migration & Miyoshi muscle dystrophy \\
PYGM & Nonsynonymous SNV & Myophosphorylase & McArdle disease \\
EXOC3L4 & Nonsynonymous SNV & Glucose and GGT activity connection & Seckel syndrome \\
SPIRE2 & Non-frameshift insertion & Intracellular transport & Osteogenesis imperfecta \\
$M E D 15$ & Nonsynonymous SNV & RNA pol II transcription & DiGeorge syndrome
\end{tabular}

SNV, single-nucleotide variant; PIP, phosphoinositol 5-phosphatase; GGT, $\gamma$-glutamyltransferase.
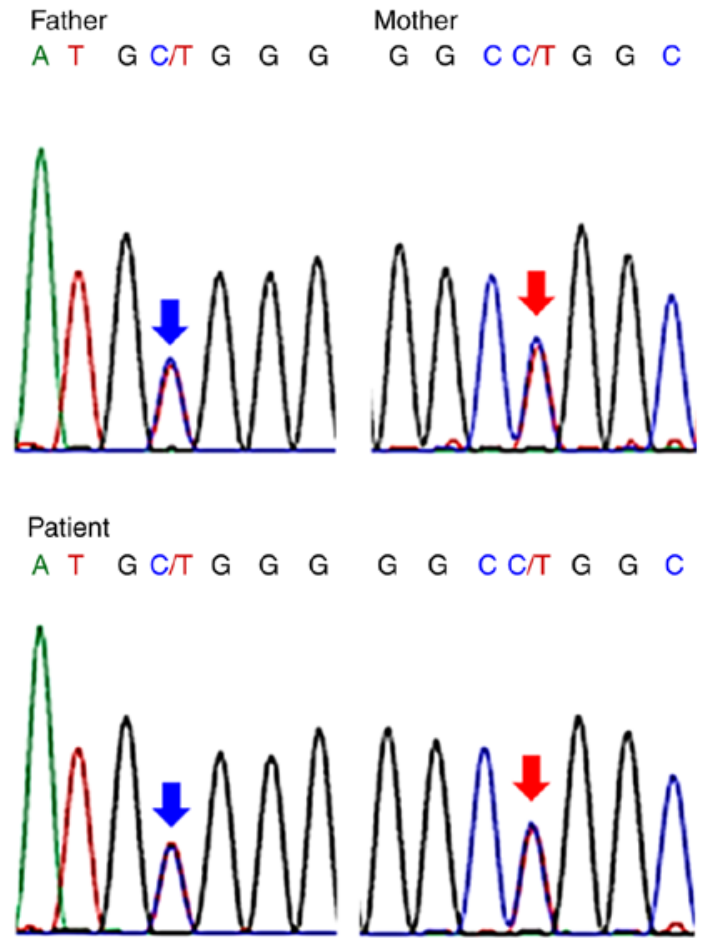

Figure 3. Sanger sequencing analysis of the patient's family with PYGM mutations. Chromatogram of the patient and her parents of the PYGM gene demonstrated that the patient have compound heterozygous mutations. The patient's father had a c.C389T (p.A130V) mutation and her mother had a c.C415T (p.R139W) mutation in exon 3.

was reduced to 28,315 , and the number was further reduced to 14,093 by filtering out synonymous SNVs. As a result of applying the recessive, compound heterozygous, and de novo conditions to the remaining 2,033 variations by filtering under the conditions of dbSNP135_common 1000G (freq >0.01), 78 de novo mutations, 12 simple recessive mutations, and 9 compound heterozygous mutations were isolated. Twelve genes with homozygous simple recessive mutations were analyzed. A detailed analysis of the function of the genes encoding the proteins and related diseases revealed that the three genes, HYAL1, LRRC17, and ZFHX3, were associated with the patient, while the remaining nine genes were found to be unrelated (Table I). Next, nine genes showing compound heterozygous mutations were analyzed. As a result, mutations in two genes, PYGM and MED15, were associated with the patient (Table II). Finally, through additional in-depth analysis, the patient's muscle weakness symptoms were predicted to be most associated with myophosphorylase dysfunction due to the $P Y G M$ mutation.

Sanger sequencing of PYGM. The exon 3 region of the PYGM gene was amplified by PCR. Primer sequences were as listed below: The first PCR, forward 5'-AAAGAACTTCAACCGGCACCC-3' and reverse 5'-TAGAAGTGCACAGGTAGCGT-3'; nested PCR, forward 5'-TGGGCCTGGCTGAGTGTTGG-3' and reverse 5'-CCAGAGATGATAAACAAGTGGG-3'. PYGM gene sequences of the patient and family were compared with the GenBank reference (AH006714.1) to confirm the mutation. The patient's father had a heterozygous mutation in the c.389C $>$ T (p.A130V), and her mother had a heterozygous mutation at c.415C >T (p.R139W). On the other hand, her sister had the same mutation as her mother. The patient had complex heterozygous mutations inherited from the father and mother, respectively (Fig. 3). As described above, the region of the PYGM gene mutation analyzed by the Sanger sequence analysis of the patient's family was exactly the same as the region in the whole-exome sequencing analysis.

Non-ischemic forearm exercise test. A non-ischemic lower arm exercise test was performed to check for changes in the intramuscular myophosphorylase enzyme due to the PYGM mutation. Anaerobic exercise increases lactic acid levels in normal subjects, but lactic acid does not increase in glycogen storage disease patients because glycogen in muscle cells cannot be used as an energy source. Following a handgrip exercise, in which a tourniquet for blood pressure measurement was closed on the hand, grasped by the hand, and then decompressed, venous blood was collected, and lactate and ammonia concentrations were measured. As a result, the concentration of ammonia in the blood ( $0 \mathrm{~min}, 11 \mu \mathrm{mol} / 1$; $1 \mathrm{~min}, 33 \mu \mathrm{mol} / \mathrm{l}$; $2 \mathrm{~min}, 31 \mu \mathrm{mol} / \mathrm{l})$ rapidly increased within $1 \mathrm{~min}$, whereas the concentration of lactate $(0 \mathrm{~min}, 0.77 \mathrm{mmol} / 1 ; 1 \mathrm{~min}$, $2.11 \mathrm{mmol} / \mathrm{l}$; $2 \mathrm{~min}, 2.11 \mathrm{mmol} / \mathrm{l}$; $3 \mathrm{~min}, 2.11 \mathrm{mmol} / \mathrm{l} ; 5 \mathrm{~min}$. 


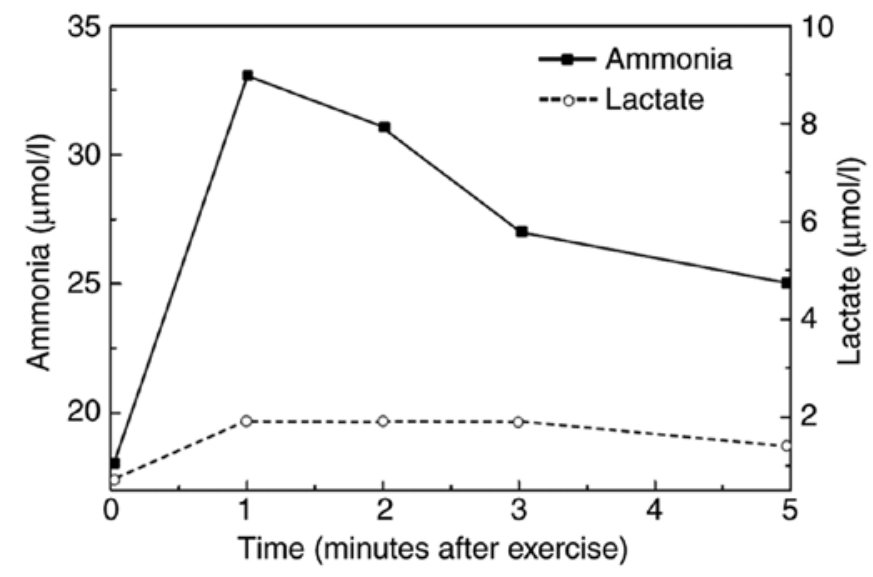

Figure 4. Ischemic forearm exercise test in a patient with McArdle disease. Following a handgrip exercise, in which a tourniquet was closed on the hand, grasped by the hand, and then decompressed, venous blood was collected, and lactate and ammonia concentrations were measured. The anaerobic forearm exercise test demonstrated no changes in venous lactate level although ammonia level increased early and sharply after exercise. The ratio between ammonia level and lactate level was higher in McArdle disease patient than that of normal person.

$1.8 \mathrm{mmol} / \mathrm{l}$ ) did not increase. The ratio of ammonia to lactate was higher than normal, which is consistent with the results of other McArdle patients (Fig. 4).

\section{Discussion}

A 13-year-old patient was diagnosed with McArdle disease via sequencing of the whole exome. The patient initially visited the growth clinic due to short stature and complained of muscle weakness. WES analysis of the patient and her family members revealed compound heterozygous mutations in the PYGM gene, which consisted of a new missense mutation and a previously reported mutation. In this patient, McArdle disease was caused by a compound heterozygous mutation of the $P Y G M$ gene, including one from her father and the other from her mother. The novel PYGM mutation in the patient, c.389C $>\mathrm{T}$, is a missense mutation of p.A130V. Another mutation in the patient, c. $415 \mathrm{C}>\mathrm{T}$, substituted amino acid arginine for tryptophan at position 139 (R139W). In two variants, the patient inherited A130V from her father and R139W from her mother, resulting in a myophosphorylase defect. The p.R139W missense mutation of the PYGM gene was reported in two Dutch male patients (15). However, the p.A130V missense mutation of the patient and her father was a new mutation that has not been reported at all.

To date, 167 variants of PYGM genes (OMIM \#608455) have been reported, and all variants can be retrieved from the LOVD database (http://database.lovd.nl/shared/variants/ PYGM). Approximately $93.4 \%$ of the PYGM mutations are in exons, and $6.6 \%$ are located in the intron regions. Most PYGM mutations are missense mutations, accounting for $50 \%$ of all mutations. Exon 1 of the $P Y G M$ gene contains the p.R50X mutation, the most common mutation among Caucasians $(16,17)$. Exon 17 is the second most variable, and the most common variant in Japanese is c.2128_2130delTTC (p.F710del) (5). The mutations found in the patient in this study, c.389 C> T and c.415 C> T, are located in exon 3.
The p.R50X mutation account for more than $50 \%$ of PYGM mutations in patients with McArdle disease in Europeans, and only one case has been reported in Japanese (5). Therefore, it was suggested that there are ethnic differences between Europeans and Asians in PYGM variation. In addition, 54\% of PYGM mutations were homozygous and the rest were heterozygous allele $(8,9)$. In one study, out of a total of fifty-four Spanish McArdle patients, $78 \%$ of the PYGM mutations were R50X and G205S (18). Further, Santalla et al (7) analyzed forty-five patients with McArdle disease in Caucasians. Among mutated alleles of PYGM, p.R50X was 55\%, whereas p.W798R and p.G205S were 10 and $9 \%$, respectively.

McArdle disease, caused by myophosphorylase deficiency, affects one in 100,000 people. Beginning in their fifties, symptoms of fixed muscle weakness appear around the shoulder and may be accompanied by cardiomyopathy, respiratory failure, and nervous system disorders $(1,11)$. In patients with McArdle disease, biopsy can be performed because of the accumulation of glycogen in skeletal muscle cells when viewed under an electron microscope. Recently, muscle biopsies have not been used to diagnose McArdle disease in children because of the burden of biopsy after general anesthesia. The exercise load test showed no false positive results, could sufficiently replace muscle biopsy, and was confirmed through sequencing of PYGM gene mutations (19). Further, de Luna et al (20) suggested that McArdle disease can be successfully diagnosed by assaying myophosphorylase in leukocytes.

Glycogen phosphorylase has three isoforms: Brain $(P Y G B)$, liver $(P Y G L)$, and muscle $(P Y G M)$. In a recent study, Llavero et al (2) showed that PYGM is present in skeletal muscle as well as in other tissues and organs, such as astrocytes, kidneys, and retinal pigment epithelium (RPE). The role of PYGM in tissues other than muscle is still unknown, but suggests the possibility of McArdle disease and comorbidities (2). There were no other comorbidities associated with PYGM mutations at the patient and family members. However, we are closely following and observing the patient and the family.

The forearm exercise test is widely used because it is useful and sensitive to diagnose muscle glycogen accumulation disorders such as McArdle disease. Diagnostic forearm exercise protocol includes ischemic and non-ischemic tests, and has high diagnostic value. In McArdle patients, myophosphorylase activity is low, resulting in a flat or blunt lactate response. In the forearm exercise test, ammonia level was used as a control, and the variation of the lactate to ammonia ratio is measured (14). As shown the results of this study, the initial increase in lactate (0-1 min) was presumed to be due to the consumption of existing glucose present in the blood. However, during the forearm exercise between $1 \mathrm{~min}$ and $5 \mathrm{~min}$, there was no increase in lactate because glycogen stored in the muscle was not used. In this study, the results of the forearm exercise test were consistent with the diagnostic results of McArdle disease patients.

Anaerobic loading does not increase blood lactate compared with a rapid increase in blood ammonia, which is often observed in patients with McArdle disease (21). In the present study, the forearm exercise test demonstrated no 
increase in lactate levels compared to a normal increase in ammonia level. Further exercise tests were not performed after five minutes to prevent muscle injury because the patient complained of muscle pain.

Next-generation sequencing (NGS) has made a number of technological advancements, with minimal validation errors as well as specificity and sensitivity at reliable levels (12). WES is a NGS method and a diagnostic approach for identifying molecular defects in patients with suspected genetic diseases. WES is used to determine the cause of genetic diseases and diagnose $25 \%$ of genetic-disease patients (13). This study is expected to contribute to the development of precision medicine in the future because NGS can be used to diagnose genetic diseases and provide accurate treatment opportunities in patients who have not been diagnosed clinically.

\section{Acknowledgements}

Not applicable.

\section{Funding}

This research was supported by the Basic Science Research Program through the National Research Foundation of Korea funded by the Ministry of Education (grant number 2017R1D1A1B03035276).

\section{Availability of data and materials}

The datasets used and/or analyzed during the current study are not publicly available due to difficulties in the complete disclosure of human genome data under Korean law but are available from the corresponding author on reasonable request.

\section{Authors' contributions}

JHK, JHP and HWB contributed to study conception and design. JSP, JHP and JHK acquired data. SKL, SHL, JHP, JHK and HWB analyzed and interpretated data. JHK, JHP and HWB drafted the manuscript. JHP, JHK and HWB critically revised the manuscript. JHK, JSP and JHP performed statistical analysis. HWB obtained funding. JHK, JHP, JSP, SKL, SHL and HWB provided administrative, technical or material support. JHK, JHP and HWB supervised the study. JHK, JHP and HWB confirm the authenticity of all the raw data. All authors read and approved the final manuscript.

\section{Ethics approval and consent to participate}

The present study was approved by the Institutional Review Board of Eulji University (approval no. EU17-42; approval date, December 14, 2017). Written informed consent was obtained from all individual participants included in this study; for participants $<18$ years old, the patients' parents provided written informed consent.

\section{Patient consent for publication}

The patient's family has provided written informed consent for publication.

\section{Competing interests}

The authors declare that they have no competing interests.

\section{References}

1. Quinlivan R, Buckley J, James M, Twist A, Ball S, Duno M, Vissing J, Bruno C, Cassandrini D, Roberts M, et al: McArdle disease: A clinical review. J Neurol Neurosurg Psychiatry 81: 1182-1188, 2010.

2. Llavero F, Arrazola Sastre A, Luque Montoro M, Gálvez P, Lacerda HM, Parada LA and Zugaza JL: McArdle disease: New insights into its underlying molecular mechanisms. Int J Mol Sci 20: 5919, 2019.

3. Lebo RV, Gorin F, Fletterick RJ, Kao FT, Cheung MC, Bruce BD and Kan YW: High-resolution chromosome sorting and DNA spot-blot analysis assign McArdle's syndrome to chromosome 11. Science 225: 57-59, 1984.

4. Nogales-Gadea G, Santalla A, Brull A, de Luna N, Lucia A and Pinós T: The pathogenomics of McArdle disease - genes, enzymes, models, and therapeutic implications. J Inherit Metab Dis 38: 221-230, 2015.

5. Sugie H, Sugie Y, Ito M, Fukuda T, Nonaka I and Igarashi Y: Genetic analysis of Japanese patients with myophosphorylase deficiency (McArdle's disease): Single-codon deletion in exon 17 is the predominant mutation. Clin Chim Acta 236: 81-86, 1995.

6. Lucia A, Ruiz JR, Santalla A, Nogales-Gadea G, Rubio JC, García-Consuegra I, Cabello A, Pérez M, Teijeira S, Vieitez I, et al: Genotypic and phenotypic features of McArdle disease: Insights from the Spanish national registry. J Neurol Neurosurg Psychiatry 83: 322-328, 2012.

7. Santalla A, Nogales-Gadea G, Encinar AB, Vieitez I, GonzálezQuintana A, Serrano-Lorenzo P, Consuegra IG, Asensio S, Ballester-Lopez A, Pintos-Morell G, et al: Genotypic and phenotypic features of all Spanish patients with McArdle disease: A 2016 update. BMC Genomics 18 (Suppl 8): 819, 2017.

8. García-Consuegra I, Rubio JC, Nogales-Gadea G, Bautista J, Jiménez S, Cabello A, Lucía A, Andreu AL, Arenas J and Martin MA: Novel mutations in patients with McArdle disease by analysis of skeletal muscle mRNA. J Med Genet 46: 198-202, 2009.

9. Nogales-Gadea G, Brull A, Santalla A, Andreu AL, Arenas J, Martín MA, Lucia A, de Luna N and Pinós T: McArdle disease: Update of reported mutations and polymorphisms in the PYGM Gene. Hum Mutat 36: 669-678, 2015.

10. Aquaron R, Bergé-Lefranc JL, Pellissier JF, Montfort MF, Mayan M, Figarella-Branger D, Coquet M, Serratrice G and Pouget J: Molecular characterization of myophosphorylase deficiency (McArdle disease) in 34 patients from Southern France: Identification of 10 new mutations. Absence of genotypephenotype correlation. Neuromuscul Disord 17: 235-241, 2007.

11. Burr ML, Roos JC and Ostör AJ: Metabolic myopathies: A guide and update for clinicians. Curr Opin Rheumatol 20: 639-647, 2008.

12. De Castro M, Johnston J and Biesecker L: Determining the prevalence of McArdle disease from gene frequency by analysis of next-generation sequencing data. Genet Med 17: 1002-1006, 2015.

13. Yang Y,Muzny DM, Reid JG, Bainbridge MN, Willis A, Ward PA, Braxton A, Beuten J, Xia F, Niu Z, et al: Clinical whole-exome sequencing for the diagnosis of mendelian disorders. N Engl J Med 369: 1502-1511, 2013.

14. Kazemi-Esfarjani P, Skomorowska E, Jensen TD, Haller RG and Vissing J: A nonischemic forearm exercise test for McArdle disease. Ann Neurol 52: 153-159, 2002.

15. Martín MA, Rubio JC, Wevers RA, Van Engelen BG, Steenbergen GC, Van Diggelen OP, De Visser M, De Die-Smulders C, Blázquez A, Andreu AL, et al: Molecular analysis of myophosphorylase deficiency in Dutch patients with McArdle's disease. Ann Hum Genet 68: 17-22, 2004.

16. Tsujino S, Shanske S and DiMauro S: Molecular genetic heterogeneity of myophosphorylase deficiency (McArdle's disease). N Engl J Med 329: 241-245, 1993.

17. Deschauer M, Morgenroth A, Joshi PR, Gläser D, Chinnery PF, Aasly J, Schreiber H, Knape M, Zierz S and Vorgerd M: Analysis of spectrum and frequencies of mutations in McArdle disease. Identification of 13 novel mutations. J Neurol 254: 797-802, 2007. 
18. Martín MA, Rubio JC, Buchbinder J, Fernández-Hojas R, del Hoyo P, Teijeira S, Gámez J, Navarro C, Fernández JM, Cabello A, et al: Molecular heterogeneity of myophosphorylase deficiency (McArdle's disease): A genotype-phenotype correlation study. Ann Neurol 50: 574-581, 2001.

19. Hogrel JY, van den Bogaart F, Ledoux I, Ollivier G, Petit F, Koujah N, Béhin A, Stojkovic T, Eymard B, Voermans N, et al: Diagnostic power of the non-ischaemic forearm exercise test in detecting glycogenosis type V. Eur J Neurol 22: 933-940, 2015.

20. de Luna N, Brull A, Lucia A, Santalla A, Garatachea N, Martí R, Andreu AL and Pinós T: PYGM expression analysis in white blood cells: A complementary tool for diagnosing McArdle disease? Neuromuscul Disord 24: 1079-1086, 2014.
21. Sinkeler SP, Daanen HA, Wevers RA, Oei TL, Joosten EM and Binkhorst RA: The relation between blood lactate and ammonia in ischemic handgrip exercise. Muscle Nerve 8: 523-527, 1985.

cc)(ㅇ) This work is licensed under a Creative Commons Attribution-NonCommercial-NoDerivatives 4.0 International (CC BY-NC-ND 4.0) License. 